ISSN 0102-261X

\title{
LAND SURFACE TEMPERATURE RETRIEVAL FROM THE THERMAL RADIANCE COLLECTED BY LANDSAT 8 TIRS SENSOR: APPLICATIONS OF FIELD AND LABORATORY MEASUREMENTS
}

\author{
RECUPERAÇÃO DE TEMPERATURA DE SUPERFÍCIE TERRESTRE DA RADIÂNCIA \\ TERMAL COLETADA PELO SENSOR TIRS/LANDSAT 8: APLICAÇÕES \\ DE MEDIDAS DE CAMPO E LABORATÓRIO
}

\author{
Pâmela Suélen Käfer \\ Advisor: Dra. Silvia Beatriz Alves Rolim (UFRGS) \\ 92 p. - Master Dissertation - 2019
}

\begin{abstract}
Land surface temperature (LST) is an important parameter in the investigation of environmental and climatic changes at various scales. However, estimating this parameter from the radiation emitted in the thermal infrared (TIR) region is a difficult task because the radiation measured by the satellite sensors is strongly affected by atmospheric effects. All LST retrieval methods require validation with field measurements. Nonetheless, the validation of this type of data is a challenge because the LST changes rapidly in time and the measurements must be performed togheter with the sensor overpass. In addition, most methodologies are developed and tested focusing on the Northern Hemisphere. Considering that operational ways of obtaining LST should be constantly investigated, the aim of this paper was to study the effect of the use of temperature-based laboratory measurements in the determination of the emissivity and LST retrieval from orbital remote sensing data. Moreover, it was intended to perform a comparative analysis among the most recent single-channel algorithms available on the literature, applied to band $10(10.6-11.19 \mu \mathrm{m})$ of the Landsat 8 TIRS. The algorithms considered were: Singlechannel generalized (SC), Improved Single-channel (ISC) and Improved Mono-window (IMW). A field of coastal dunes was chosen as study area. Two sets of laboratory emissivity measurements were performed with field samples at different temperatures using a Fourier Transform Infrared (FT-IR). Emissivity and temperature data were obtained in the study area concomitantly with the satellite overpass. The Radiative Transfer Equation (RTE) with parameters of global atmospheric profiles was tested as a method of validation. A variation of approximately $2 \%$ in the emissivity in relation to the temperature was observed, which could be neglected. The FT-IR presents limitations on the period to acquire stability, however as long as this limitation is respected and the calibration approach correctly carried out, laboratory measurements can achieve optimum accuracy and replace field validation. Available spectral libraries of emissivity have also proved to be a good alternative. All evaluated single-channel methods are suitable for obtaining LST; however, ISC provided superior results in all analyzes, producing higher $\mathrm{R}^{2}(0.99978)$ and lower RMSE (0.019) relative to the other algorithms tested.
\end{abstract}

RESUMO. A temperatura da superfície terrestre (Land surface temperature - LST) é um importante parâmetro na investigação de mudanças ambientais e climáticas em várias escalas. Entretanto, estimar esse parâmetro da radiação emitida na região do infravermelho termal (TIR) é uma tarefa difícil, pois as radiações medidas pelos sensores dos satélites são fortemente afetadas por efeitos atmosféricos. Todos métodos de recuperação de LST requerem validação com medidas de campo. Porém, a validação deste tipo de dado é um desafio, visto que a LST muda rapidamente no tempo e as medidas devem ser realizadas em conjunto com a passagem do sensor. Além disso, a maioria das metodologias são desenvolvidas e testadas com foco no hemisfério norte. Tendo em vista que maneiras operacionais de se obter LST devem ser constantemente investigadas, o objetivo desta pesquisa foi estudar o efeito do uso de medidas de emissividade de laboratório tomadas com base em temperaturas na determinação da LST a partir de dados de sensoriamento remoto orbital. Ademais, pretendeu-se realizar uma análise comparativa entre os algoritmos single-channel mais recentes existentes na literatura, aplicados à banda $10(10,6-11,19 \mu \mathrm{m})$ do Landsat 8 TIRS. Os algoritmos considerados foram: Single-Channel Generalizado (SCG), Improved Single-Channel (ISC) e Improved Mono-Window (IMW). Um campo de dunas costeiras foi escolhido como área de estudo. Dois conjuntos de medidas de emissividade de laboratório foram construídos com amostras de campo em diferentes temperaturas com uso de um Fourier Transform Infrared (FT-IR). Dados de emissividade e temperatura foram obtidos na área de estudo concomitamente com a passagem do sensor. A equação de transferência radiativa (Radiative Transfer Equation - RTE) com parâmetros de perfis atmosféricos globais foi testada como forma de validação de dados. Uma variação de aproximadamente $2 \%$ na emissividade em relação à temperatura foi observada, podendo ser negligenciada. O FT-IR apresenta limitações quanto ao período para adquirir estabilidade, porém respeitando esta limitação e realizando abordagem correta de calibração, medidas laboratoriais podem atingir ótima acurácia e substituir a validação de campo. Bibliotecas espectrais disponíveis de emissividade demonstraram ser também uma alternativa válida. Todos os métodos single-channel avaliados são adequados para obter LST; no entanto, o ISC forneceu resultados superiores em todas as análises, produzindo maior $\mathrm{R}^{2}(0,99978)$ e menor RMSE (0.019) em relação aos demais. 